\title{
A complex structure in the mRNA of Tf1 is recognized and cleaved to generate the primer of reverse transcription
}

\author{
Jia-Hwei Lin and Henry L. Levin ${ }^{1}$ \\ Laboratory of Eukaryotic Gene Regulation, National Institutes of Child Health and Human Development, \\ Bethesda, Maryland 20892 USA
}

\begin{abstract}
All retroviruses and LTR-containing retrotransposons are thought to require specific tRNA molecules to serve as primers of reverse transcription. An exception is the LTR-containing retrotransposon Tf1, isolated from Schizosaccharomyces pombe. Instead of requiring a tRNA, the reverse transcriptase of Tf1 uses the first 11 bases of the Tf1 transcript as the primer for reverse transcription. The primer is generated by a cleavage that occurs between bases 11 and 12 of the Tf1 mRNA. Sequence analysis of the $5^{\prime}$ untranslated region of the Tf1 mRNA resulted in the identification of a region with the potential to form an RNA structure of 89 bases that included the primer binding site and the first 11 bases of the Tf1 mRNA. Systematic mutagenesis of this region revealed 34 single-point mutants in the structure that resulted in reduced transposition activity. The defects in transposition correlated with reduced level of $\mathrm{Tf} 1$ reverse transcripts as determined by DNA blot analysis. Evidence that the RNA structure did form in vivo included the result that strains with second site mutations that restored complementarity resulted in increased levels of reverse transcripts and Tf1 transposition. The majority of the mutants defective for reverse transcription were unable to cleave the Tf1 mRNA between bases 11 and 12. These data indicate that formation of an extensive RNA structure was required for the cleavage reaction that generated the primer for $\mathrm{Tf} 1$ reverse transcription.
\end{abstract}

[Key Words: Priming; retrotransposon; RNA; RT; S. pombe; Tfl]

Received September 20, 1996; revised version accepted December 2, 1996.

Retroviruses and long terminal repeat (LTR)-containing retrotransposons proliferate in hosts that comprise the full spectrum of eukaryotic evolution. Although the LTR-containing retroelements propagate in organisms as diverse as yeasts and mammals, the mechanisms of reverse transcription are well conserved (Gabriel and Boeke 1993; Leis et al. 1993). To initiate replication, retroviruses and LTR-containing retrotransposons require specific tRNA molecules to serve as primers. The $3^{\prime}$ terminal 10-18 bases of the tRNA anneal to the transcript of the element at a region adjacent to the 5' LTR called the primer binding site (PBS). The element-encoded reverse transcriptase uses the $3^{\prime} \mathrm{OH}$ provided by the tRNA to initiate DNA synthesis of the first product of reverse transcription, the minus-strand strong-stop DNA (-ssDNA) (Varmus and Swanstrom 1984; Kikuchi et al. 1986; Chapman et al. 1992). All LTR-containing retroelements are thought to require tRNA for priming because the elements that have been sequenced have a PBS that is complementary to known tRNA species. An interesting exception is Tf 1 , an LTR-containing retro-

${ }^{1}$ Corresponding author.

E-MAIL Henry_Levin@nih.gov; FAX (301) 496-0243. transposon isolated from Schizosaccharomyces pombe (Levin et al. 1990).

Tf1 is a highly active transposon that encodes Gag, protease (PR), reverse transcriptase (RT), and integrase (IN) proteins within a single open reading frame (ORF). The PBS sequence of Tfl has no complementarity to any known tRNA species, suggesting that no tRNA molecules are able to serve as the primer for $\mathrm{Tf} 1$ reverse transcription. Instead, our laboratory has proposed that Tfl undergoes a unique mechanism of self-primed reverse transcription. We noted that perfect complementarity exists between the first 11 nucleotides of the Tf1 mRNA and its PBS. On the basis of this observation and the results of a mutagenesis study, it was proposed that the first 11 bases of the Tf1 transcript fold back and anneal to the PBS to serve as primer (Levin 1995). Data from this previous report showed that in vivo transposition activity and the accumulation of -ssDNA in Tfl particles was greatly reduced as the result of mutations in the PBS or the first 11 nucleotides of the Tf1 mRNA (Levin 1995). Compensatory mutants that restored complementarity between these sequences rescued both the transposition activity and the ability to synthesize the -ssDNA. In addition, recent data from our laboratory demonstrate that the Tf1 mRNA is cleaved between 
bases 11 and 12 in a reaction that requires the RNase $H$ domain of the Tf1 RT (Levin 1996). These results suggested that the first 11 bases of the Tf1 transcript anneal to the PBS and serve as the primer for reverse transcription.

Because the replication of retroviruses begins with the annealing of a cellular tRNA to the PBS, interactions between the primer tRNA and the viral mRNA were studied extensively to understand how retroviruses initiate reverse transcription. In addition to the annealing of the 3' end of a tRNA to the PBS, two other regions of the tRNA have been found to interact with retroviral transcripts (Aiyar et al. 1992, 1994). Formation of a binary complex through extended interactions between the tRNA primer and the PBS of the retrotransposon Tyl have also been found to be an essential step in initiating reverse transcription (Wilhelm et al. 1994; Keeney et al. 1995; Friant et al. 1996). In light of the extensive interactions between viral RNA and tRNA that are required for the initiation of retroviral reverse transcription, we conducted a detailed analysis of the $5^{\prime}$-untranslated region (5' UTR) in the Tf1 mRNA. We identified a sequence in this region that had the potential to form an RNA structure of 89 bases that included the primer binding site and the first 11 bases of the Tf1 mRNA (Fig. 1A). This complex structure includes a hairpin and three duplexes formed by long-range nucleotide interactions. We systematically mutated the Tf 1 sequences in the $5^{\prime}$ UTR to test the contribution of this sequence to Tfl transpo- sition and to determine whether the putative RNA structure can form in vivo. The results of this study indicated that the complex RNA structure encompassing the PBS was required for the cleavage reaction that generated the primer of $\mathrm{Tf} 1$ reverse transcription.

\section{Results \\ Sequence analysis of the 5' UTR of Tf1 and Tf2 $m R N A$}

Sequence analysis of the $5^{\prime}$ UTR in the Tf1 mRNA revealed that 89 nucleotides had the potential to fold into a structure that included the PBS. The proposed RNA structure is presented in Figure 1A. This structure includes a hairpin and three duplexes formed by long-range nucleotide interactions. In addition to the PBS duplex reported previously (Levin 1995), we identified sequence that could potentially form a 5-bp duplex upstream and an 11-bp duplex downstream of the PBS duplex. The 5-bp duplex and the PBS duplex are separated by a bulge of 4 nucleotides in the top strand. Adjacent to the 11-bp duplex, a hairpin with the potential to form a 12-bp stem and a 6-nucleotide loop was also found. Base position 1 in Figure 1A is the first nucleotide of Tf1 mRNA as expressed from the Tf 1 promoter.

Sequence with the potential to form a similar RNA structure was also found in $\mathrm{Tf} 2$, a retrotransposon closely related to $\mathrm{Tfl}$ that was also isolated from $S$.

B
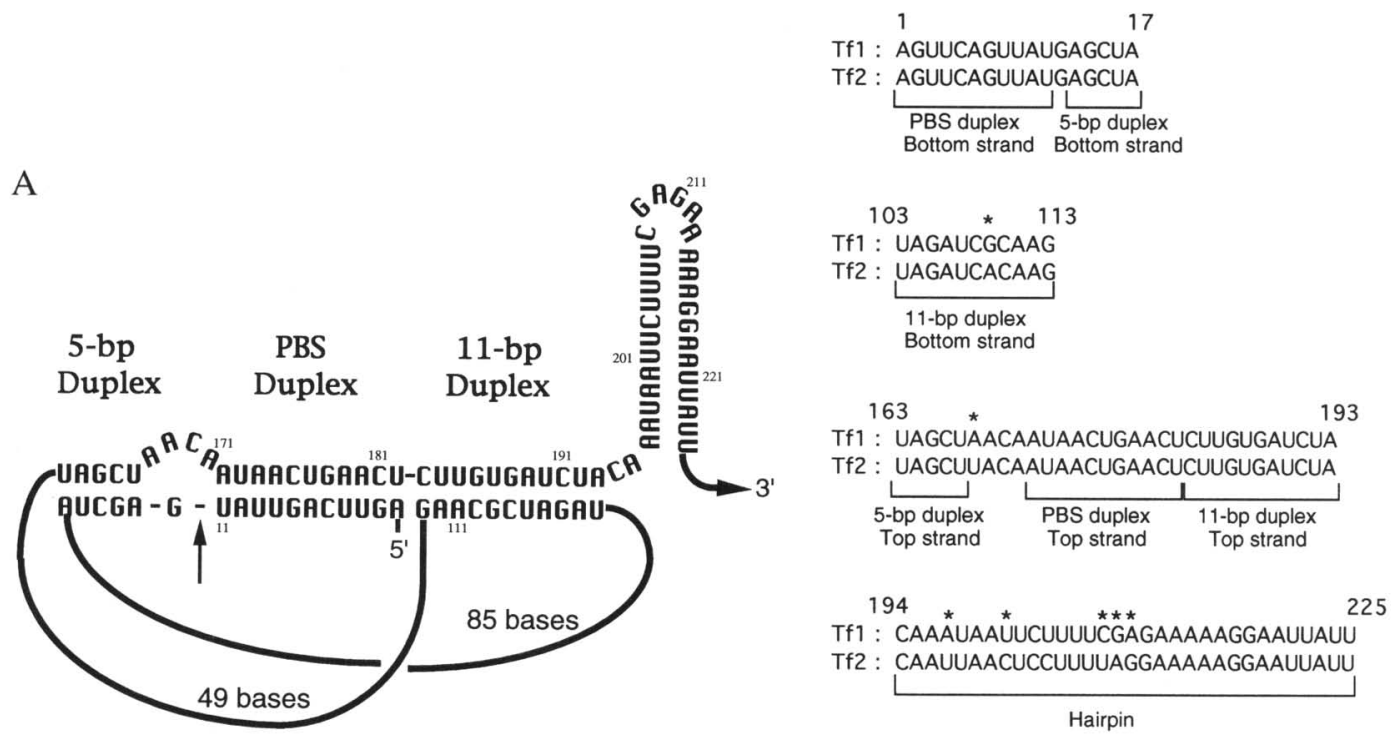

Figure 1. The proposed RNA structure in the 5' UTR of the Tf 1 mRNA. (A) The sequence of the three duplexes and the hairpin. The $5^{\prime}$ end of the mRNA is indicated, and the relationship to the 3' end of the mRNA is shown. The vertical arrow indicates the position of the cleavage that creates the primer. The names of the duplexes are indicated. Every tenth nucleotide position is indicated relative to the first nucleotide of the Tf1 mRNA as expressed from the Tf1 promoter. The length of the loops between the duplexes are indicated. $(B)$ Sequences in the RNA structure are well conserved between Tf 1 and Tf 2 . The number above each duplex termini indicates the position of the nucleotide in the Tf 1 mRNA. The differences in sequences between Tf 1 and Tf 2 are marked with asterisks $(*)$. 
pombe (Levin et al. 1990). Although Tf1 and Tf2 have significantly divergent gag sequences, nucleotides included in the RNA structure were found to be well conserved (see Fig. 1B). The putative RNA structure of Tf2 differs slightly from that of $T f 1$ in the hairpin. The two additional differences between $\mathrm{Tf} 1$ and $\mathrm{Tf} 2$ include a position in the bulge where Tf 2 has a $U$ instead of an A and a position in the bottom strand of the 11-bp duplex where Tf2 has an A instead of a G. The latter change allows Tf2 to form an A:U base pair instead of the G:U base pair in the Tfl counterpart.

\section{Screening a library of random mutations in the $5^{\prime}$} UTR of Tf1 for changes that affect Tf1 transposition

More than one-fourth of the nucleotides (89 of 353) in the Tf $15^{\prime}$ UTR are involved in the formation of this complex structure that included the PBS of Tf1. On the basis of this observation and the results of a previous study from our laboratory supporting a model of selfprimed reverse transcription, we speculated that this putative RNA structure may participate in the initiation of Tf1 reverse transcription.

To evaluate the role of the putative RNA structure in Tf 1 transposition, we conducted a mutagenesis study of the $5^{\prime}$ UTR. We generated a library of Tf 1 plasmids that contained single-point mutations in a region of $\mathrm{Tf} 1$ that included the putative structure. This plasmid library was then screened for mutations that caused transposition defects. The method used to measure transposition was based on the resistance to G418 that cells acquire as the result of the transposition of a neo-marked copy of $\mathrm{Tf} 1$ from the ura3-containing plasmid into the genome. Figure 2 shows the assay plasmid with its copy of Tf 1 that can be induced because the $T f 1-n e o$ element was fused to the $n m t 1$ promoter that is activated when cells are grown in the absence of thiamine. After induction, patches of cells were transferred to a plate with medium that contained $5^{\prime}$-fluoro-orotic acid (5-FOA), a drug that selects against the presence of the ura3 gene and therefore against the presence of the plasmid. These patches were then replica printed to a YES 5-FOA-G418 plate to identify cells that contained copies of Tf1-neo that had transposed into the genome.

Figure 2 illustrates how the library was generated. We first created a XhoI-SpeI cassette in the Tf1 expression plasmid to facilitate the cloning of the mutagenized fragment. This XhoI-SpeI cassette version of the Tfl plasmid (pHL891-19) produced wild-type levels of $\mathrm{Tfl}$ transposition when transformed into $S$. pombe (data not shown). We therefore used this plasmid for the wild-type Tfl element in the following study. The region containing the putative structure in the $5^{\prime}$ UTR is expanded in Figure 2 to indicate which regions of complementarity were subjected to the mutagenesis. We produced mutations throughout the sequence in oligonucleotides HL100 and HL101 by creating degenerate pools of these oligonucleotides that were used to amplify this fragment by PCR. The addition of all four nucleotides during the oligo synthesis at a concentration 250 -fold less than the normal

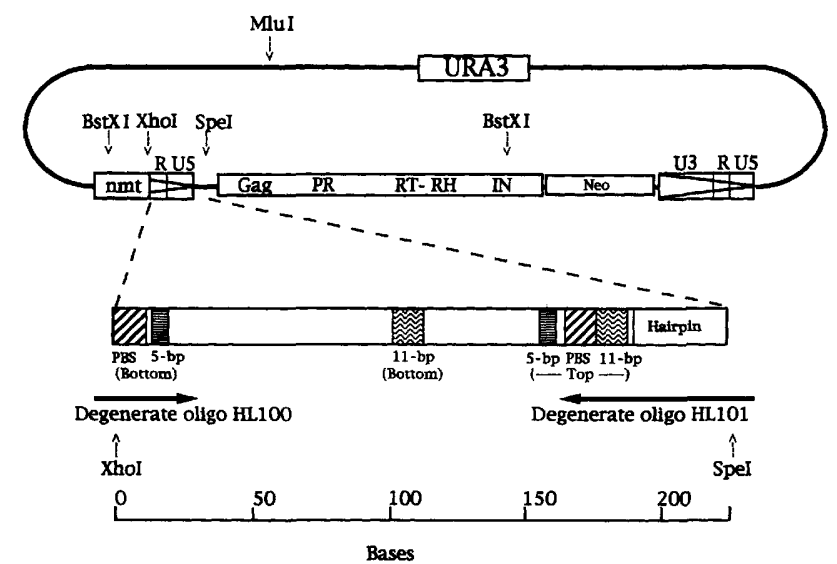

Figure 2. The structure of the Tf1-containing plasmid and the mutagenesis of the $5^{\prime}$ UTR. The sequence of Tf 1 includes two direct LTRs and a single ORF that encodes capsid protein Gag, protease ( $P R)$, reverse transcriptase (RT), and integrase (IN). The LTRs are depicted by open wedges on either side of the ORF (indicated by a rectangle that is labeled with the name of the proteins encoded). The U3, R, and U5 regions of the LTRs are indicated. The box labeled nmt represents the $n m t 1$ promoter that replaces the U3 region in the 5' LTR; the box labeled neo is the inserted neo gene, and the box labeled URA3 is the URA3 gene of $S$. cerevisiae. The restriction sites in the plasmid used in the experiments are indicated. The area with the potential to form an RNA structure in the $5^{\prime}$ UTR is expanded, and the regions of complementarity are indicated by the boxes with the same pattern of shading. Each pattern is labeled according to which duplex and which strand is represented. The lengths of the two degenerate oligonucleotides, HL100 and HL101, are represented by the horizontal arrows with the arrowheads indicating the $3^{\prime}$ end of the oligonucleotides.

level ensured that the majority of the plasmids contained no more than a single-point mutation. The two degenerate oligonucleotides included most of the bases in the putative structure except nucleotides 103-113 and 163167. Assuming that all four nucleotides are incorporated with equal probability, we predicted that $70.6 \%$ of the plasmids would have wild-type sequence, $24.6 \%$ of the plasmids would have single-point mutations, and $4.8 \%$ of the plasmids would contain multiple-point mutations. The degenerate PCR fragment was ligated into the Xhol and SpeI sites of pHL891-19, and the resulting 11,350 bacterial transformants obtained from electroporation were amplified and pooled for library plasmid isolation.

We transformed the mutant plasmid library into $S$. pombe and used the transposition assay to screen for transformants that showed reduced transposition activity in vivo. The results of the mutant screening are summarized in Table 1. Among 5000 transformants screened, 384 mutants $(7.68 \%)$ were found to have reduced transposition activity as determined by the patch assay. One fourth of the defective transformants were randomly chosen to be extracted for plasmid isolation, and the nucleotides between the XhoI and SpeI sites were sequenced. Fifty-eight plasmids were found to con- 
Table 1. Genetic screen for strains defective in transposition

S. pombe transformants screened 5000

Transformants defective in Tf1 transposition

Plasmids rescued from defective transformants

Plasmids sequenced to identify the mutation

Plasmids with different single point mutations

Transposition phenotypes reconfirmed

Tramsformants with normal expression of Tf1 proteins

tain base changes that represented 37 unique mutations. The sequence between the MluI (see Fig. 2) and the SpeI sites from all 37 plasmids was subcloned into a wild-type copy of the same vector to confirm that the Tf1 sequence outside of the XhoI-SpeI cassette was wild type. We then tested the expression of $\mathrm{Tf} 1$ proteins by immunoblot analysis of Gag and IN proteins. Thirty-four mutant plasmids were found to express normal levels of Tfl proteins.

The single-point mutations that affect Tf1 transposition clustered within the putative RNA structure

Figure 3 shows the sequence subjected to mutagenesis and the distribution of the single-point mutants in the RNA structure. Represented in uppercase letters in the RNA structure are sequences subjected to mutagenesis, and letters bordering the structure are the mutations. All base changes were found to cluster within the complementary regions of the proposed RNA structure. We identified 6 mutations in the PBS and 10 mutations in the bottom strand of the PBS duplex. We also found four mutations in the bottom strand of the 5-bp duplex. The most noticeable result is the presence of the 14 mutations in the top strand of the 11-bp duplex. Not a single mutation was found in the non-base-pairing regions (nucleotides 12, 18-30, and 170-171) that were subjected to mutagenesis. The only mutant found in the hairpin created a start codon by converting AAG to AUG. This base change resulted in generating an additional ORF upstream of Gag such that the translation of $\mathrm{Tfl}$ proteins was inhibited.

\section{Targeted mutagenesis of the putative RNA structure}

We explored specific aspects of the RNA structure by generating targeted mutations at eight distinct positions. The positions and the transposition effect of these sitedirected mutations are indicated in Figure 3 by nucleotides marked with an asterisk. Judging from the patch assay results, the transposition defects due to mutations U11A and G14C appeared severe and were comparable to those produced by mutations in the same regions, Al0G and $\mathrm{C} 15 \mathrm{~A}$. To characterize duplex sequence not subjected to mutagenesis, three site-directed mutations were generated in the bottom strand of the 11-bp duplex and one was created in the top strand of the 5-bp duplex. All four mutants resulted in significantly reduced Tf1 transposition. We also created two site-directed point mutations, $\mathrm{C} 170 \mathrm{G}$ and $\mathrm{A} 171 \mathrm{C}$, in the bulge region between the PBS and the 5-bp duplexes. Both changes drastically reduced $\mathrm{Tf} 1$ transposition activity. Because these two mutations were in the last two nucleotides of the U5 region, the base changes were likely to result in lower transposition because of the change in the IN binding site on the reverse transcripts.

\section{The quantitative measurement of transposition defects}

The magnitude of the transposition defects caused by each mutation was measured by subjecting each strain to a quantitative transposition assay. In a method similar to the transposition patch assay described above, cells on

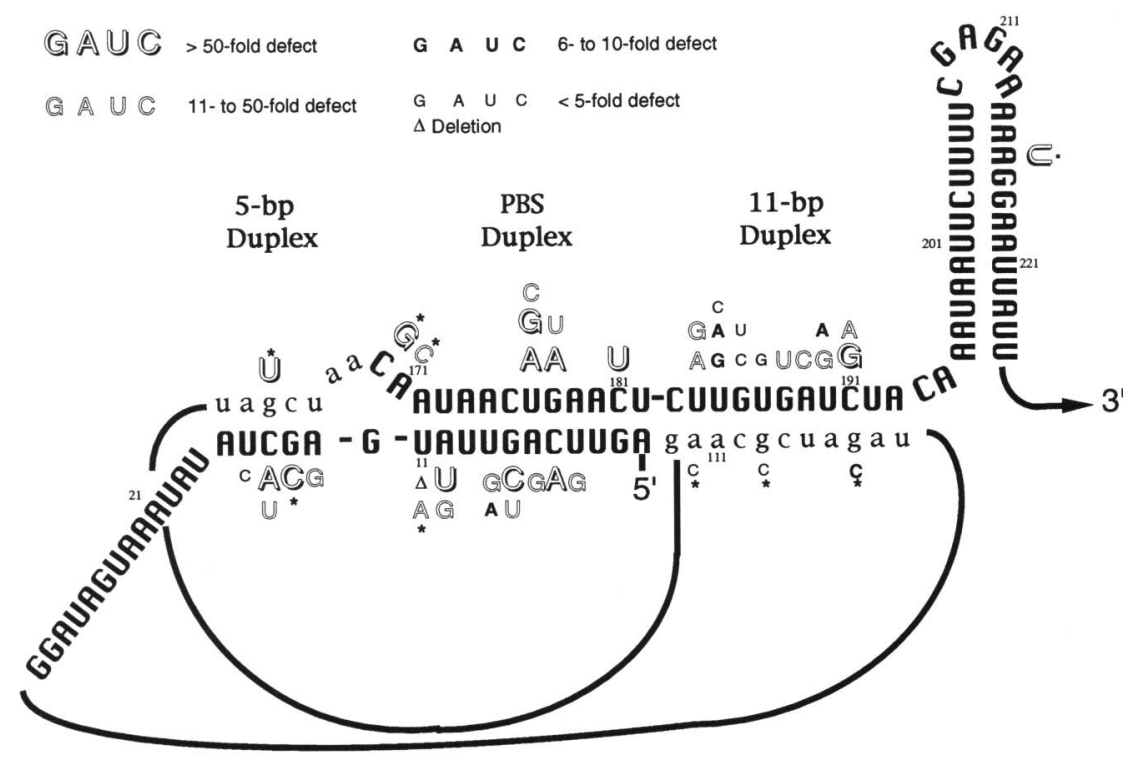

Figure 3. The distribution of single-point mutations in the putative RNA structure. The uppercase letters in the RNA structure are sequences that were subjected to random mutagenesis, and the letters bordering the structure represent the mutations that caused transposition defects. Eight site-directed mutations are denoted by asterisks $(*)$. The mutation A216T, $(\cdot)$ was found to inhibit the production of Tfl proteins. The magnitude of the transposition defects are indicated by the size of the lettering. 
agar plates were grown without thiamine to induce transposition and were then resuspended in liquid media. By spreading cells on 5-FOA containing plates at a density that produced $\sim 5000$ colonies, we could detect the fraction of induced cells that acquired genomic copies of Tf1-neo by replica printing the 5-FOA-resistant colonies to 5-FOA-G418 plates. Table 2 is the list of the actual and relative transposition frequencies of all single-point mutants with transposition defects. The actual transposition frequency from each strain was obtained by averaging the measurements from at least three independent patches of cells. The relative transposition frequency was normalized to that of the wild-type strain and listed as \%WT. The 100-fold drop in the frequency of G418-resistant colonies because of the frameshift in IN indicated that the level of recombination events that were independent of transposition was low. The results of the quantitative transposition assays are also indicated in Figure 3 by the sizes and symbols of the nucleotides. Mutations in the PBS and 5-bp duplexes were more likely to cause severe defects as only 3 of the 25 changes in these duplexes had transposition frequencies $\geqslant 10 \%$ of wild type. In comparison, 11 of the 17 mutations in the 11-bp duplex resulted in frequencies $\geqslant 10 \%$ of wild type. The potential of forming G:U base pairs existed in the mutants A6G, A10G, A13G, C15U, and $\mathrm{Cl} 181 \mathrm{U}$. Two of these mutants, A10G and C15U, resulted in transposition frequencies two- to threefold higher than other base change at the same position. These results suggested that at least the A10G and C15U mutations, and possibly the others, allowed the formation of duplexes that contained $\mathrm{G}: \mathrm{U}$ base pairs.

\section{The detection of base-pairing interactions within the 5' UTR of the Tf1 mRNA}

The hypothesis that the complementary strands of the duplexes anneal in vivo was tested by measuring the transposition of Tf1 elements that contained pairs of mutations predicted to restore base-pairing and therefore rescue transposition. Previously published results from this type of analysis produced strong evidence that nucleotides within the PBS base-pair with the first 11 bases of the Tf1 mRNA (Levin 1995). We conducted the same type of study on the 5- and 11-bp duplexes. As seen in Figure 4A, the transposition defects of all three top strand mutants (U184G, U187G, and C191G) and all three bottom strand mutants (A112C, G109C, and $\mathrm{G} 105 \mathrm{C}$ ) in the 11-bp duplex were rescued at least partially by strains with compensating mutations that restored complementarity. The mutations $\mathrm{A} 112 \mathrm{C}$ and U184G resulted in transposition frequencies of $36.8 \%$ and $13.5 \%$ of wild type, respectively. The combination of these two changes rescued the level of transposition to the remarkable level of $166 \%$ of wild type, indicating that the transposition did dependent on the level of stability generated by hydrogen bonding between these two bases. The mutation $\mathrm{U} 187 \mathrm{G}$, which converted a potential G:U pair to $\mathrm{G}: \mathrm{G}$ mismatch, caused only a minor defect in Tf1 transposition and transposed at $70.1 \%$ of wild-type frequency. The mutation G109C, on the opposite strand of $\mathrm{U} 187 \mathrm{G}$ in the putative structure, resulted in a transposition frequency of $31.8 \%$ of wild type. The combination of these two mutations, U187G/G109C, partially rescued the transposition defects caused by the G109C mutant and exhibited a transposition frequency of $75.1 \%$ of wild type. The mutation C191G resulted in the most severe transposition defect in the 11-bp duplex and had a transposition frequency of only $0.67 \%$ of wild type. The mutation on the opposite strand, G105C, resulted in $10 \%$ of wild-type transposition frequency. The combined double point mutant, G105C/C191G, restored Tf 1 transposition to $44.7 \%$ of the wild-type frequency. This represented at least a 4 -fold rescue above the activity of the single mutation G105C and a 66-fold rescue above the activity of the $\mathrm{C} 191 \mathrm{G}$ mutation. These results demonstrated that nucleotide interactions exist between the two strands of the 11-bp duplex.

The increase in $\mathrm{Tf} 1$ transposition caused by combining two detrimental mutations that reestablish complementarity was also observed with mutations in the 5-bp duplex (Fig. 4B). The two single-point mutations on the opposite strands of the 5-bp duplex, C15A (bottom strand) and G165U (top strand), both greatly reduced the Tf1 transposition activity and resulted in transposition frequencies of $0.82 \%$ and $0.86 \%$ of wild type, respectively. The combined mutations, C15A/G165U, resulted in $13.92 \%$ of the wild-type transposition frequency that represented a 16-fold rescue of $\mathrm{Tf} 1$ transposition activity. The absence of total rescue could be attributable to the possibility that both the formation of this complex structure and the sequences within the structure are important for Tfl transposition.

Sequence alterations in two upstream short ORFs and the putative hairpin exhibit no reduction in $T f 1$ transposition activity

An interesting feature that exists in the Tfl mRNA is the presence of two short ORFs located at least 260 nucleotides upstream of the initiation codon of the major Tf1 ORF. A similar feature was found in the mRNA of Rous sarcoma virus (RSV), which contains three ORFs in the mRNA leader upstream of the AUG initiator of the gag gene. These three ORFs were found to contribute to viral protein translation, mRNA packaging, and viral infectivity (Donze and Spahr 1992; Moustakas et al. 1993a,b). The initiation codons of the two short ORFs in Tf1 were removed (mutations G12A and G25C), and no effect on transposition was observed (data not shown). Thus, the transposition defects caused by other mutations found in the vicinity of the short ORFs were not attributable to effects on translation of these two short peptides.

The role of the putative hairpin (nucleotides 196-225) in the transposition mechanism remained unclear because the solitary mutation in the hairpin, A216T, created an ORF that resulted in decreased translation of the Gag and Pol proteins (data not shown). To analyze further the function of the putative hairpin, more severe 
Table 2. List of transposition frequencies of all single-point mutants

\begin{tabular}{|c|c|c|c|c|}
\hline \multirow{2}{*}{$\begin{array}{l}\text { Mutation } \\
\mathrm{U} 4 \mathrm{G}\end{array}$} & \multicolumn{2}{|c|}{$\begin{array}{l}\text { Actual transposition } \\
\text { frequency }(\%)^{\mathrm{a}}\end{array}$} & \multirow{2}{*}{$\begin{array}{c}\begin{array}{c}\text { Relative transposition } \\
\text { frequency }(\% \mathrm{WT})\end{array} \\
6.40\end{array}$} & \multirow[t]{2}{*}{ Region } \\
\hline & $0.243 \pm 0.065$ & (4) & & \\
\hline C5A & $0.007 \pm 0.012$ & (4) & 0.22 & \\
\hline A6G & $0.279 \pm 0.154$ & $(3)$ & 6.54 & \\
\hline G7C & $0.059 \pm 0.038$ & (4) & 1.37 & \\
\hline G7U & $0.156 \pm 0.175$ & (4) & 3.64 & \\
\hline U8G & $0.114 \pm 0.087$ & (4) & 3.54 & bottom strand \\
\hline U8A & $0.403 \pm 0.099$ & (4) & 12.55 & of PBS duplex \\
\hline A10U & $0.058 \pm 0.052$ & (4) & 1.82 & \\
\hline $\mathrm{A} 10 \mathrm{G}$ & $0.156 \pm 0.142$ & (4) & 4.87 & \\
\hline U11A & $0.103 \pm 0.036$ & (3) & 2.49 & \\
\hline $\mathrm{U} 11 \Delta$ & $1.076 \pm 0.306$ & (3) & 26.22 & \\
\hline $\mathrm{A} 13 \mathrm{G}$ & $0.138 \pm 0.047$ & (4) & 3.23 & \\
\hline G14C & $0.049 \pm 0.046$ & (3) & 1.18 & bottom strand \\
\hline C15A & $0.035 \pm 0.024$ & (4) & 0.82 & of 5-bp duplex \\
\hline $\mathrm{C} 15 \mathrm{U}$ & $0.078 \pm 0.054$ & (4) & 2.42 & \\
\hline $\mathrm{U16C}$ & $0.846 \pm 0.280$ & (4) & 26.34 & \\
\hline G105C & $0.340 \pm 0.130$ & $|3|$ & 10.00 & bottom strand \\
\hline G109C & $0.944 \pm 0.085$ & (3) & 31.80 & of 11-bp duplex \\
\hline Al12C & $1.120 \pm 0.165$ & $(3)$ & 36.80 & \\
\hline G165U & $0.024 \pm 0.034$ & $|3|$ & 0.86 & $\begin{array}{l}\text { top strand of } \\
5 \text {-bp duplex }\end{array}$ \\
\hline $\mathrm{Cl} 170 \mathrm{G}$ & $0.024 \pm 0.033$ & $(3)$ & 0.86 & bulge \\
\hline A171C & $0.102 \pm 0.054$ & $(3)$ & 3.68 & Duige \\
\hline U177A & $0.044 \pm 0.029$ & (4) & 1.36 & \\
\hline U177G & $0.080 \pm 0.060$ & (4) & 1.87 & \\
\hline $\mathrm{U} 177 \mathrm{C}$ & $0.188 \pm 0.046$ & (4) & 4.40 & top strand of \\
\hline G178A & $0.054 \pm 0.034$ & (4) & 1.28 & PBS duplex \\
\hline G178U & $0.095 \pm 0.078$ & (4) & 2.96 & \\
\hline $\mathrm{C} 181 \mathrm{U}$ & $0.054 \pm 0.076$ & $|3|$ & 1.32 & \\
\hline U184A & $0.118 \pm 0.082$ & (4) & 2.75 & \\
\hline U184G & $0.310 \pm 0.059$ & (7) & 10.09 & \\
\hline U185A & $0.475 \pm 0.063$ & (4) & 11.14 & \\
\hline U185G & $0.471 \pm 0.072$ & (4) & 11.05 & \\
\hline $\mathrm{U} 185 \mathrm{C}$ & $0.740 \pm 0.254$ & (4) & 23.02 & \\
\hline $\mathrm{G} 186 \mathrm{C}$ & $1.071 \pm 0.270$ & (4) & 25.11 & top strand of \\
\hline G186U & $1.175 \pm 0.191$ & (3) & 28.62 & 11-bp duplex \\
\hline U187G & $2.465 \pm 0.229$ & (7) & 78.91 & \\
\hline G188U & $0.173 \pm 0.066$ & (4) & 5.38 & \\
\hline $\mathrm{A} 189 \mathrm{C}$ & $0.197 \pm 0.039$ & (4) & 4.63 & \\
\hline U190G & $0.225 \pm 0.093$ & (4) & 7.00 & \\
\hline U190A & $0.636 \pm 0.249$ & (4) & 14.91 & \\
\hline C191G & $0.026 \pm 0.023$ & (7) & 0.67 & \\
\hline C191A & $0.164 \pm 0.037$ & (4) & 5.09 & \\
\hline IN-fs & $0.030 \pm 0.021$ & (12) & 0.95 & \\
\hline PR-fs & $<0.001 \pm 0.001$ & (6) & $<0.02$ & \\
\hline WT & $3.471 \pm 0.672$ & (21) & & \\
\hline
\end{tabular}

${ }^{a}$ Transposition frequencies were obtained by averaging at least three measurements from each strain and expressed as the average \pm S.D. The number of measurements are indicated in the parentheses.

mutations were made that included changing the stretch of purines (nucleotides 209-213) to pyrimidines, changing the stretch of pyrimidines (nucleotides 202-207) to purines, and deleting the hairpin (nucleotides 196-225) altogether. None of these changes had any effect on Tf1 transposition. 
A

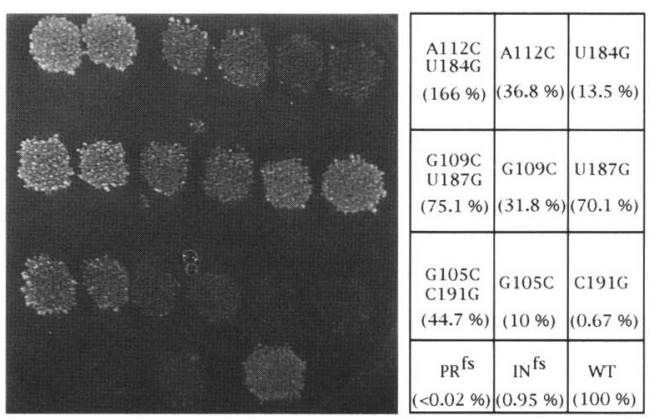

B
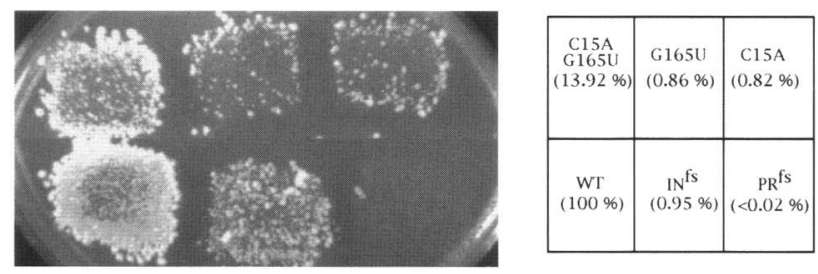

Figure 4. Transposition assay results showing that defects caused by single-point mutations in the 11- and 5-bp duplexes were restored by the addition of mutations that reestablished complementarity. (A) Results of transposition assays of strains with mutations in the 11-bp duplex. Pairs of independent yeast transformants that contained mutant versions of Tf1 were induced for transposition and printed onto an 5-FOA plate, which was then printed onto the 5-FOA-G418 plate shown. The level of growth indicated the frequency of transposition. The sequence of the base changes is indicated, and the results of quantitative transposition assays (as percent of wild type) are shown in parentheses. $(B)$ Results of transposition assays of strains with mutations in the 5-bp duplex. The mutations in each strain are indicated with the corresponding positions on the boxes at the right. Numbers in the parentheses indicate the results of quantitative transposition assays represented in transposition frequency relative to wild type level.

\section{Mutations that disrupt the RNA structure affect the synthesis of $T f 1$ reverse transcripts}

Because the RNA structure contained the PBS, we speculated that the bulk of the mutations that reduced transposition frequencies specifically reduced the priming of reverse transcription. To test this hypothesis, we used DNA blot analysis to measure the levels of the reverse transcripts (cDNA) synthesized by strains that contained these mutations (Fig. 5). Levels of the double-stranded reverse transcripts of Tfl can be determined by performing DNA blot analysis on total $S$. pombe DNA extracts digested with BstXI (Atwood et al. 1996). A 2.1-kb cDNA band derived from the reverse transcript of $\mathrm{Tf} 1-n e o$ can be detected using a radiolabeled neo fragment as probe. In the same DNA blot, the $9.5-\mathrm{kb}$ plasmid band can also be visualized. The majority of the mutant strains produced either very little or no detectable Tf1 reverse transcript, whereas the levels of the plasmid bands detected were comparable to that of wild type. All mutants in the
PBS produced no detectable 2.1 - kb signal. In addition, no signal was detected in the extracts made from the strains with mutations in the bottom strand of the PBS duplex, except for the strains that contained mutations U8A and U11 $\Delta$. These two mutations resulted in transposition frequencies of $12.5 \%$ and $26.22 \%$ of wild type, respec-

A
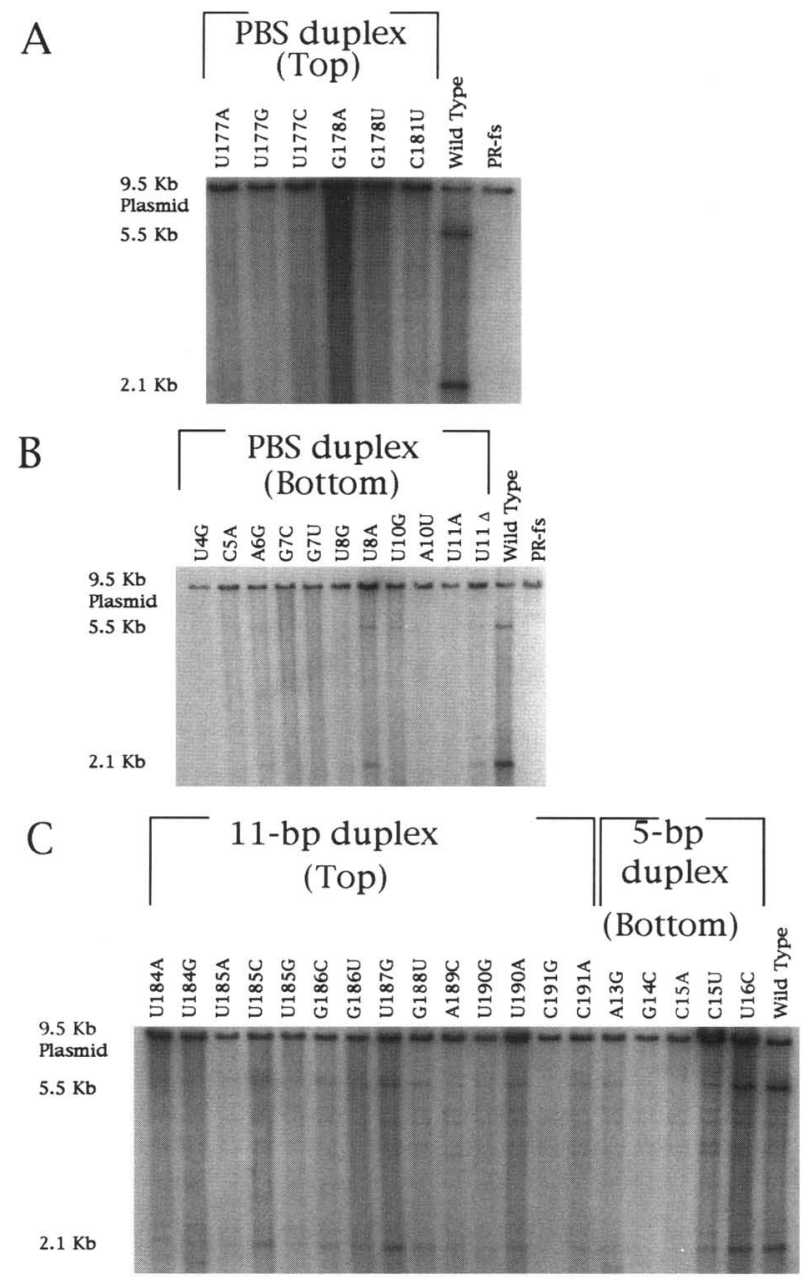

Figure 5. DNA blots showing reduced levels of cDNA produced by versions of Tfl with mutations in the $5^{\prime}$ UTR. Total DNA was extracted from induced cells grown to stationary phase and digested with $B s t \mathrm{XI}$. The filter was probed with a $1-\mathrm{kb}$ neo fragment. (A) A blot of DNA from strains with mutations located in the PBS. The label at the top of each lane indicates the mutation of the strain from which the DNA was extracted. The wild-type strain was the positive control and the PR-fs, the strain with a frameshift in $\mathrm{PR}$, expressed no RT and was the negative control. The $9.5-\mathrm{kb}$ signal was the plasmid copy of Tfl, and the $2.1 \mathrm{~kb}$ signal was a cDNA fragment of Tf1. The $5.5-\mathrm{kb}$ signal was a single LTR-containing cDNA fragment whose structure was not confirmed. $(B)$ A blot of DNA from strains with mutations located in the first 11 bases of the Tf 1 mRNA. All descriptions in this blot are the same as in $A$. (C) A blot of DNA from strains with mutations located in the top strand of the 11-bp duplex and the bottom strand of the 5-bp duplex. All descriptions in this blot are the same as in $A$. 
tively, which reflected much higher levels than were caused by the other mutations in the same region.

The levels of the $2.1-\mathrm{kb}$ signal produced by all strains with mutations in the top strand of the 11 -bp duplex also correlated with the transposition frequencies detected. The majority of the mutants in this region expressed reduced but visible $2.1-\mathrm{kb}$ bands with the exception of the two most severe mutants, U184A and C191G, which produced very faint signal. These two mutations also generated AUG initiation codons and resulted in 3- to 7-fold lower transposition frequencies than those of other changes at the same positions. Although these mutations had no effect on the expression of Tfl proteins, it was possible that translational factors bound to these AUGs and prevented these nucleotides from interacting with the nucleotides in the bottom strand of the 11-bp duplex. Data from mutants on the bottom strand of 5-bp duplex showed similar results. Four of five mutants in this region produced little or no $\mathrm{Tf} 1$ reverse transcript. However, an easily visible $2.1-\mathrm{kb}$ band was detected in the strain that contained the U16C mutation, which resulted in a transposition frequency of $26.34 \%$ of wild type. These results indicated that the reduced transposition frequencies caused by the single-point mutations correlated well with and may be attributable to the reduced levels of Tfl reverse transcripts.

We described above the rescue of defective Tfl elements that resulted from combining two mutations that restored the complementarity of the duplexes. We tested the possibility that the restoration of the RNA structure that resulted in increased Tf1 transposition caused increased reverse transcription. The DNA blot in Figure 6 indicated that copies of $\mathrm{Tf} 1$ with pairs of mutations that re-established complementarity in all three duplexes generated an increase in the amount of reverse tran- scripts. The intensities of the $2.1-\mathrm{kb}$ band produced by the three strains that contained compensating pairs of mutations in the 11-bp duplex were much stronger than those produced by the strains with single-point mutations. Two previously reported sets of compensating mutants (Levin 1995) in the PBS duplex also produced significantly higher levels of $\mathrm{Tfl}$ reverse transcripts than the individual point mutants (Fig. 6). This result is consistent with the previously reported transposition frequencies of $80 \%$ of wild type produced by the strains with the compensating pairs of mutations in the PBS duplex. The strain that contained a pair of compensating mutations in the 5-bp duplex produced significantly more cDNA than the strains that contained the individual point mutations. In summary, we detected higher levels of reverse transcripts produced by versions of $\mathrm{Tf} 1$ that contained compensating pairs of mutations than by copies of Tf1 that contained the corresponding singlepoint mutations.

The levels of reverse transcripts synthesized in all mutant strains were quantified by comparing the intensities of the $2.1-\mathrm{kb}$ cDNA bands to those of the $9.5-\mathrm{kb}$ plasmid bands using PhosphorImaging analysis. To examine the relationship between the magnitude of the transposition defects caused by the mutations and the levels of reverse transcripts that resulted, we plotted transposition frequencies against relative cDNA levels detected for each mutant strain. We found that the transposition frequencies of the mutant strains were directly related to the amount of cDNA by a second-order relationship (Fig. 7). This correlation suggested that the majority of the mutants were defective in transposition because of a defect in the production of $\mathrm{Tf} 1$ reverse transcripts.

We showed above that two site-directed mutants in the bulge region between the PBS and the 5-bp duplexes,

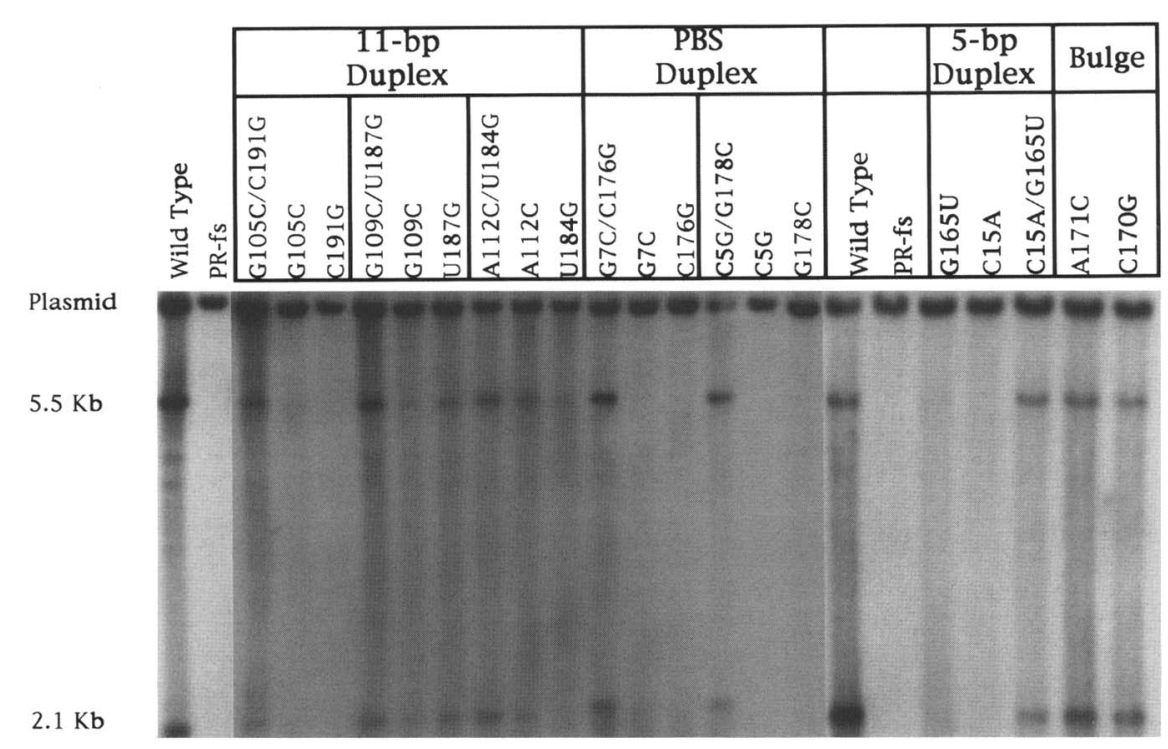

Figure 6. A DNA blot showing increased levels of cDNA produced by strains with mutations that restored complementarity within the RNA structure. The preparation and the detection of the DNA in this blot were the same as described in Fig. 5. The wild-type and the PR-fs strains served as the positive and the negative controls for reverse transcription, respectively. The 9.5-kb signal was the plasmid copy of $\mathrm{Tf} 1$, and the $2.1-\mathrm{kb}$ signal was a cDNA fragment of Tfl. The lanes on the blot are labeled in groups of three representing two strains with single-point mutations opposite each other in the duplexes and one strain with the combined double mutations that restored complementarity. The strains in the 11- and 5-bp duplexes were strains constructed for this study. The strains in the PBS duplex were strains constructed previously (Levin 1995). The strains with the mutations located in the bulge, $\mathrm{C} 170 \mathrm{G}$ and $\mathrm{A} 171 \mathrm{C}$, are also indicated. 


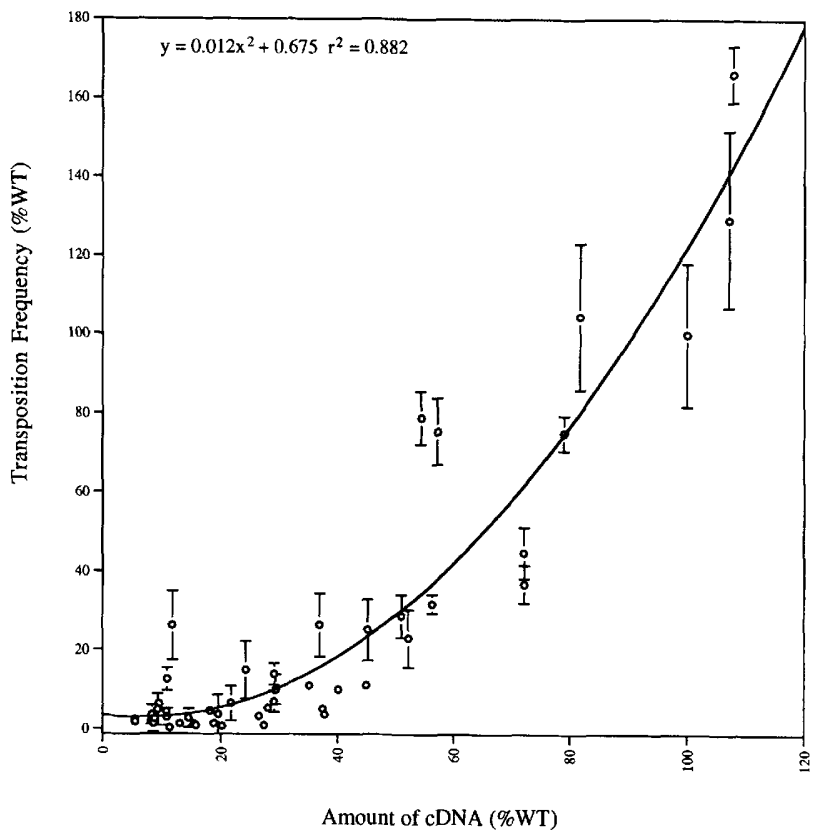

Figure 7. A plot of transposition frequency vs. the amount of cDNA produced by each mutant strain. Each strain was subjected to a quantitative transposition assay, and the actual transposition frequency was obtained by averaging the measurements from at least three independent assays. The transposition frequency was normalized to that of the wild-type strain (represented as \%WT). The amount of cDNA produced by each strain was measured quantitatively by comparing the intensity of the $2.1-\mathrm{kb}$ band to the $9.5-\mathrm{kb}$ plasmid band in the DNA blot using PhosphorImaging analysis. The amount of cDNA produced was also normalized to that produced by the wild-type strain. Data obtained from strains with single-point mutations, double-point mutations, and mutations in the ORFs were included in this plot. The relationship between the transposition frequency and the amount of cDNA produced could be approximated by a second-order equation listed at the top of the plot in which $x$ represents the amount of cDNA and $y$ represents the transposition frequency. The error bars indicates the standard deviation of the relative transposition frequency.

C170G and A171C, were defective in Tf1 transposition. We speculated that the transposition defects could be attributable in part to the changes in nucleotides that IN may bind during integration. If this is the case, these two mutations should not affect the reverse transcription of Tf1. Quantitative analysis of the $2.1-\mathrm{kb}$ signal produced by these two strains, C170G and $\mathrm{Al71C}$, revealed that these mutations caused a 2.8- and a 3.3-fold defect in reverse transcription, respectively. These data indicated that sequence in the bulge also contribute to the reverse transcription mechanism even in the case where the duplexes are preserved.

\section{Detection of -ssDNA}

In a previous report (Levin 1995), strains that contained versions of Tfl with single-point mutations in the PBS duplex accumulated significantly less -ssDNA than the wild-type strain. The addition of compensating mutations that reestablished hydrogen bonding in the duplex resulted in wild-type levels of -ssDNA. Two species of -ssDNAs were detected in wild-type strains when a minus-strand-specific oligonucleotide was used as a probe (Levin 1995). The difference in electrophoretic mobility between the two species of -ssDNAs produced by wildtype Tf 1 was found to be attributable to the absence of -11 nucleotides of sequence at the $3^{\prime}$ end of the shorter species. Together with our current understanding that the first 11 bases of the mRNA are removed (Levin 1996), we proposed that the shorter strong-stop species was the result of the reverse transcription of Tf 1 mRNA that was cleaved between bases 11 and 12. To establish whether the mutations in this study affected the levels of -ssDNA, we tested a representative sample of strains with single-point mutations in different regions of the RNA structure for any defect in the synthesis of -ssDNA. No -ssDNA could be seen in samples extracted from strains that contained mutations A10G, U11 1 , and U11A in the bottom strand of the PBS duplex and the mutation C191G in the top strand of the 11-bp duplex (Fig. 8). The strain that contained Tfl with the mutation U16C was the only mutant that produced clearly visible amounts of -ssDNA, albeit more of the shorter species was detected than the full-length DNA. In each of these cases, the levels of -ssDNA produced correlated well with the levels of $2.1-\mathrm{kb}$ cDNA signal observed and the frequencies of transposition detected.

\section{The effect of mutations in the RNA structure on the levels of primer cleavage}

Thus far, nine separate point mutations in the $5^{\prime}$ end of Tf1 mRNA [four from previous work (Levin 1995) and five from this study] resulted in low levels of -ssDNA. This provides strong support for the model that formation of this structure is required for priming of reverse transcription. Recent results from our laboratory (Levin

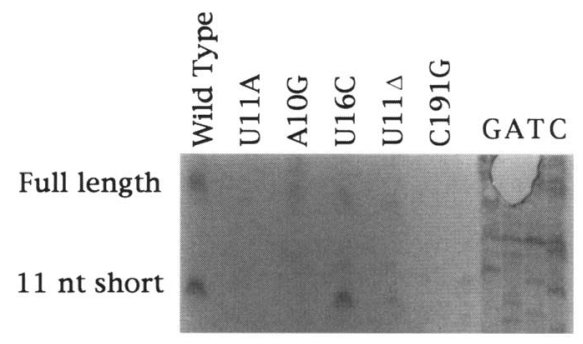

Figure 8. A DNA blot showing the effect of mutations in the RNA structure on the levels of -ssDNA. Nucleic acid extracted from virus-like particles was analyzed by DNA blot hybridization analysis. The minus-strand strong-stop species of DNA were detected using JB341, a radiolabeled oligonucleotide probe that was specific for the minus strand. The sequencing ladders were generated using kinased HL78 as a primer, the oligonucleotide that hybridized to the PBS (Levin 1995). Two species of -ssDNAs, the full-length and the DNA that was 11 bases shorter, were produced by the wild-type strain. 
1996) showed that the primer for synthesizing -ssDNA is generated by a cleavage of the Tf1 mRNA between bases 11 and 12. A technique that was developed to measure the levels of cleaved Tfl mRNA was reported (Levin 1996). The assay included primer extension reactions to detect the levels of Tf1 mRNA that was cleaved at base 11. As a way to improve the sensitivity of the assay, a mutation was incorporated in the polymerase active site of Tfl RT to prevent DNA polymerization and the subsequent degradation of the cleaved Tfl mRNA that is catalyzed by RNase H. Aspartic acid 564 (Because the PR cleavage sites that define the amino and carboxyl termini of Tf1 RT have not been precisely mapped, the number refers to the position within the primary translation product) in Tfl reverse transcriptase is the second $\mathrm{D}$ in the highly conserved RT motif YMDD and when it is changed to asparagine the polymerase activity of RT is eliminated. The result of the D564N mutation is the increased accumulation of Tf 1 mRNA cleaved between bases 11 and 12. Here we used this technique to determine whether any of the mutations in the RNA structure result in reduced levels of cleaved primer. Small amounts of cleaved Tf1 mRNAs were detected in samples extracted from either log-phase or stationaryphase cells of wild-type strains as seen in Figure 9. We observed no increase in cleaved Tf 1 mRNA from stationary-phase cells because the cleaved Tf 1 mRNA intermediates were reverse transcribed and then degraded by RNase $H$. As expected, we detected a substantial increase in the level of cleaved Tf 1 mRNA in a strain with the mutation D564N. The D564N mutation was then cloned into the versions of Tf 1 with mutations in the $5^{\prime}$ UTR to test the effect of base changes in the RNA structure on primer cleavage. Primer extension reactions were conducted on DNA samples extracted from stationaryphase cells of strains with these double mutations. Results of these primer extension reactions are shown in Figure 9.

Most strains with mutations in the RNA structure were either defective or inefficient in cleaving off the first 11 nucleotides of Tf 1 mRNA. The intensity of primer extension products were also quantified using
PhosphorImaging analysis. The fraction of Tf1 RNA cleaved was calculated by dividing the intensity of the shorter primer extension product by the combined intensity of both full-length and shorter primer extension products. These values were represented in parentheses in Figure 9 as percent primer cleavage. Because we detected-ssDNA produced by the strain that contained the mutation U16C and this strain transposed at $26 \%$ of wild-type frequency, we were not surprised to observe that it also produced cleaved Tf 1 mRNA. Strains that contained either of two mutations that generated a GU pair, A10G and A13G, showed close to half of wild-type primer cleavage, indicating the formation of a weaker interaction allowed some primer cleavage. We were intrigued to find that the strain with the mutation U11A produced nearly wild-type levels of the cleaved mRNA even though this strain generated no detectable -ssDNA or $2.1 \mathrm{~kb}$ reverse transcription product (Fig. 8). A likely explanation for this result is that the mismatch between A11 and A172 did not affect the formation of the overall RNA structure or the primer cleavage but it prevented the extension of the primer.

\section{Discussion}

Our examination of the sequence in the $5^{\prime}$ UTR of Tfl revealed four regions with the potential to form RNA base pairs within an 89-nucleotide structure composed of three duplexes and a hairpin. We found the sequences included in these putative interactions were well conserved between Tf1 and Tf2, and in both cases they included the PBS. Results from both random and site-directed mutagenesis showed that the mutations that caused defective transposition clustered in sequences that had the potential to form duplex interactions.

Evidence that the regions of complementarity in the RNA did fold into duplex structures derived from the observation that the transposition defects caused by individual mutations could be rescued if point mutations were combined such that the complementarity of the duplex was restored. The base-pairing in the PBS duplex was demonstrated in a paper published previously (Levin

Figure 9. Primer extension analysis of total RNA showing the effect of mutations on the levels of the cleaved Tf1 mRNA. The RNA analyzed came from strains that contained wild-type or mutant versions of Tf1 RT. The flanking sequencing ladders were generated using kinased JB890, the same oligonucleotide that was used in the primer extension reactions. (Arrow a) the primer extension products templated by cleaved Tf1 mRNA; (arrow b) the primer extension products templated by fulllength Tfl mRNA. The top band (indicated by arrow $c$ ) is a nonspecific product that is also present in strains without Tfl/data not shown). (WT log) RNA samples isolated from log-phase cells; (WT stat) RNA samples isolated from stationary-phase cells. All other samples were isolated from stationary-phase cells of strains with the D564N mutation only or in combination with mutations in the 5' UTR. Numbers in parentheses indicate the percentage of the total Tfl signal in the lane that was templated by the cleaved mRNA. 
1995). In the results described above, we found that the transposition defects caused by single-point mutations at three positions of the 11-bp duplex and one position of the 5-bp duplex could be rescued by additional mutations that restored complementarity. These results strongly suggested that the formation of the three duplexes play an important role in the Tfl transposition pathway. It is interesting to note that alternative RNA structures other than the one we proposed could occur if two separate molecules of Tf1 RNA participate in the formation of the RNA duplexes.

The result that we could not detect any effect on Tf 1 transposition of mutations in the hairpin is puzzling. It is possible that in our system the replacement of the original $\mathrm{Tf} 1$ promoter with the $n m t 1$ promoter may have bypassed a requirement for the hairpin related to transcription. Alternatively, growth conditions in the laboratory may not adequately mimic specific conditions in the wild that could affect the function of the hairpin. When a Tf 1 transposition assay was conducted at room temperature instead of at $32^{\circ} \mathrm{C}$, the strains that contained the hairpin deletion transposed at a slightly lower rate than the wild type (data not shown). This result suggested that the levels of Tf1 transposition could be affected by the hairpin when cells grow in the wild.

The mutations that caused lower levels of transposition also produced reduced amounts of cDNA without affecting Tfl protein expression. Mutations that re-established the complementarity of the altered duplexes also caused an increase in the production of cDNA. These results indicated that the complex RNA structure was required for the completion of reverse transcription.

Quantitative transposition assays revealed that the magnitude of the transposition defects caused by the mutations correlated well with the amounts of $\mathrm{Tfl}$ cDNA produced by these strains. When transposition activity was plotted against cDNA levels, the position of the points was best approximated by a function of the square of the cDNA concentration. The shape of this curve, as seen in Figure 7, indicated that a threshold level of cDNA was required for efficient transposition. The accumulation of a basal amount of reverse transcripts might be required to saturate nonproductive interactions with cellular factors. An alternative explanation for the shape of this curve is that the rate limiting step could be cooperatively regulated.

The low levels of -ssDNA produced by representative strains that contained point mutations in various regions of the 5' UTR supported our model that formation of the RNA structure was required for the priming of reverse transcription. The results of primer extension assays designed to measure the amount of the cleaved Tf1 mRNA show that the majority of the mutations blocked the cleavage and indicated that the complex RNA structure was required for this step in the synthesis of -ssDNA. The result that the U11A mutation caused reduced levels of -ssDNA without a significant reduction in the level of cleaved mRNA provided strong evidence that the first 11 bases of the mRNA did serve as primer. This conclusion is based on the prediction that the UllA base change would cause a mismatch at the $3^{\prime}$ end of the primer that would inhibit synthesis of -ssDNA.

One puzzling result is that the strain with the mutation U187G showed near wild-type levels of transposition and reverse transcription though it produced no primer cleavage under our assay conditions. Because our recent genetic data suggested that the Tf1 RNase $\mathrm{H}$ domain is required to cleave the mRNA (Levin 1996), one possibility is that the mutation in the polymerase active site of RT used in our cleavage assay rendered the enzyme unable to recognize and cleave the modified RNA structure that resulted from U187G mutation, whereas the wild-type RT was able to cleave the modified mRNA and complete reverse transcription. This suggested that the polymerase active site of RT may recognize and bind to the 11-bp duplex before the cleavage can occur. To initiate DNA synthesis, the RT must either reorientate the position of its active site relative to the cleavage site or allow an additional RT molecule to start polymerization.

Base-pairing between the nucleotides in the PBS and the 5-bp duplexes resulted in the formation of a bulge between the two duplexes. We speculated that the formation of this bulge structure opposite the cut site is required for cleavage because it resulted in a strained structure that destabilized the phosphodiester bond between bases 11 and 12 . Alternatively, the bulge might be positioned opposite the cleavage site to provide the active site of a nuclease with access to the phosphodiester bond that is cleaved. In either case, the identity of the nucleotide at positions 11 and 12 must not be critical, as the U11A and G12A mutations resulted in near wildtype levels of cleavage. Biochemical and X-ray crystallographic studies of HIV RT (Kohlstaedt et al. 1992) showed that the distance between the polymerase active site and the RNase $\mathrm{H}$ active site of RT is $\sim 18-20 \mathrm{bp}$. If Tf1 RT possesses a similar structure, the binding of the polymerase active site of Tf1 RT to the 11-bp duplex would locate the active site of RNase $\mathrm{H}$ near the bulge and allow for the primer cleavage to occur.

Although we have presented evidence that indicates the sequence in the $5^{\prime}$ UTR of the Tf 1 message formed a complex RNA structure that is specifically recognized and cleaved by a nuclease to generate the primer for reverse transcription, we cannot exclude the possibility that some of our point mutations may result in inefficient packaging of the Tfl mRNA into virus-like particles (VLPs). If this is true, lower levels of Tf1 mRNA would be exposed to the enzymes in the VLPs such as RT that may be required for the cleavage reaction. This would suggest that some regions of the complex RNA structure could be required for RNA packaging. To address this issue, we have made several efforts to develop a packaging assay to measure the levels of Tfl mRNA in VLPs. Measuring packaged mRNA has been particularly difficult in the case of all retrotransposons because their VLPs are not secreted into culture medium. Thus far, we have been unable to purify $\mathrm{Tf} 1$ particles away from the bulk of the cellular transcripts and therefore cannot measure any defects in packaging of Tfl mRNA. 
Extended contacts of the primer tRNA with the sequences flanking the PBS of RSV and Tyl have been identified through in vivo and in vitro studies. The formation of individual duplexes contributes to the efficient annealing and packaging of the tRNA primers. $\mathrm{Mu}$ tations resulting in the disruption of these interactions caused reduced propagation of RSV and Tyl (Wilhelm et al. 1994; Keeney et al. 1995; Friant et al. 1996). In addition, the results of in vitro reverse transcription indicated that mutations in the Tyl structure resulted in low levels of annealed tRNA (Wilhelm et al. 1994). Three short regions of Tyl RNA downstream of the PBS, named boxes 0,1 , and 2.1 , interact with the $T$ and $D$ stems and loops of tRNA ${ }_{i}^{\text {met }}$ (Fig. 10), respectively. In the case of the RSV, the structures that form as the result of base-pairing of the mRNA include the U5-IR stem and the U5-leader stem (Fig. 10). The structures that form between the mRNA and the tRNA ${ }^{\text {Trp }}$ include the PBS duplex and a 7-base sequence upstream of the PBS that interacts with $\mathrm{T} \psi \mathrm{C}$ arm of tRNA ${ }^{\mathrm{Trp}}$ (Fig. 10). The formation of a large RNA structure and a 4- or 5-base bulge near the PBS bears similarity to the structure of $\mathrm{Tfl}$ (Fig. 1).

The structural and functional similarities of these extended RNA complexes suggest that RTs of retroelements recognize and bind to a specific RNA conforma-

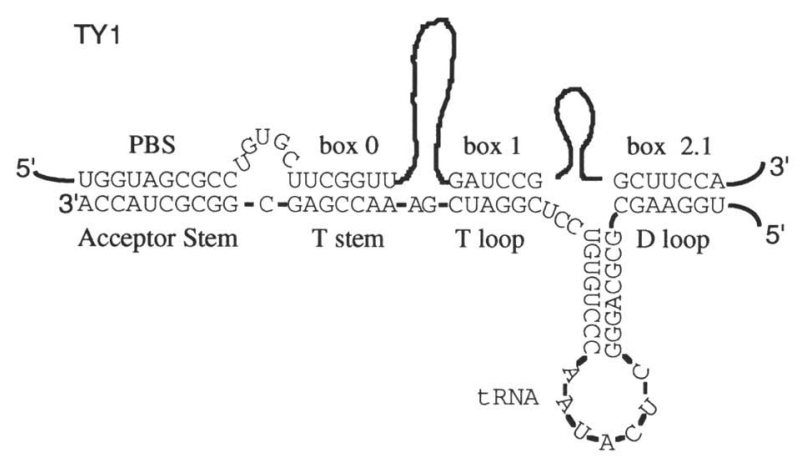

RSV

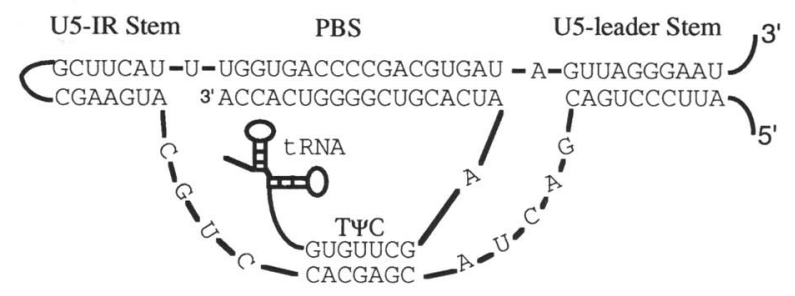

Figure 10. The binary RNA complexes of RSV and Ty1 formed with primer tRNA. (Top) The structure of Tyl as reported by Friant et al. (1996). Interactions among PBS and regions termed box 0 , box 1 , and box 2.1 of Ty1 RNA with acceptor stem, T stem, $\mathrm{T}$ loop, and D loop of $\mathrm{tRNA}^{\mathrm{Met}}$ are indicated. (Bottom) The structure of RSV as reported by Aiyar et al. (1994). Formation of U5-IR and U5-leader stems by RSV mRNA are indicated. Interactions between the acceptor stem and $\mathrm{T} \psi \mathrm{C}$ arm of the tRNA $^{\text {Trp }}$ with the PBS and a sequence in the U5 region of RSV are shown. tion to initiate reverse transcription. Interestingly, the mechanism of Tfl cleavage and self-priming bares resemblance to the mechanism of plus-strand priming from the polypurine tracts of retroviruses and LTR-containing retrotransposons. Both mechanisms require $\mathrm{RNase} \mathrm{H}$ to generate the primer for reverse transcription. It is possible that the recognition of the polypurine tract by $\mathrm{RNase} \mathrm{H}$ and the production of the Tfl primer require similar features yet to be discovered.

\section{Materials and methods}

Media

The $S$. pombe minimal liquid and plate media were composed of Edinburgh minimal medium (EMM) (Moreno et al. 1991) and were supplemented with amino acids, vitamin B1, 5-FOA and Geneticin (G418) as described previously (Levin 1996).

\section{Transposition assay}

$S$. pombe strains with $\mathrm{Tf} 1$ plasmids were first grown as patches on EMM in the absence of Vitamin B1 to induce the nmt1 promoter that was fused to the neo-marked Tf1. After 4 days of $32^{\circ} \mathrm{C}$ incubation, the plates were replica printed to EMM medium containing 5-FOA to select against cells containing the Tfl-neo plasmid (Boeke et al. 1987). These plates were then replica printed to YES medium (Moreno et al. 1991) containing 5-FOA-G418 and incubated at $32^{\circ} \mathrm{C}$ for 2 days to detect cells with copies of Tf1-neo that had transposed into the genome (Levin and Boeke 1992; Levin et al. 1993).

The actual transposition frequency of each mutant strain was determined with a quantitative transposition assay (Levin 1995) and was normalized to the frequencies of wild-type strains assayed at the same time as control.

\section{Strains and plasmid constructions}

The yeast strains used in this paper are listed in Table 3. All plasmids were transformed into YHL912, a strain of $S$. pombe with the genotype $h^{-}$, ura4-294, and leu1-32. All plasmids were constructed by directional cloning of PCR-amplified fragments into a Tfl-containing plasmid. All PCR reactions described were performed with the native $P f u$ enzyme (Stratagene). The specific oligonucleotides used in the construction of each mutation are shown in Table 4. Restriction sites were created or abolished by fusion PCR (Levin 1995).

The mutation sites in each plasmid generated by PCR were sequenced with dideoxynucleotide termination reactions (U.S. Biochemical Sequenase v. 2.0 DNA sequencing kit) to verify the nature of the base changes. In addition, each PCR-generated construct was made in duplicate using an independent PCR reaction to control against the possibility of nontemplated PCRgenerated mutations. Each duplicated plasmid gave results in transposition and primer extension assays equivalent to that of its partner.

\section{Construction of the XhoI-SpeI cassette in the Tf1-containing} plasmid

Plasmids pHL891-19 and pHL900-1 are wild-type and PRframeshift versions of Tfl-containing plasmids, respectively, that both lacked the Xhol site in the neo marker and contained a unique SpeI site that was generated to introduce mutations in the 5' UTR of Tf1. Plasmid pHL891-19 was the wild-type Tf1 
Table 3. Yeast strains

\begin{tabular}{|c|c|c|c|c|c|c|c|}
\hline \multirow[b]{2}{*}{ Yeast strain $^{\mathrm{a}}$} & \multicolumn{3}{|c|}{ Plasmid description } & \multirow[b]{2}{*}{ Yeast strain ${ }^{a}$} & \multicolumn{3}{|c|}{ Plasmid description } \\
\hline & number & Tf1 allele & region & & number & Tf1 allele & region \\
\hline YHL4093 & pHL891-19 & WT & - & YHL5134 & pHL1142-1 & $\mathrm{U} 4 \mathrm{G}$ & PBS \\
\hline YHL4179 & pHL900-1 & PR-fs & PR & YHL5128 & pHL1139-1 & $\mathrm{C} 5 \mathrm{~A}$ & PBS \\
\hline YHL1306 & pHL431-25 & IN-fs & IN & YHL5158 & pHL1154-1 & $\mathrm{A} 6 \mathrm{G}$ & PBS \\
\hline YHL5038 & pHL1120-1 & hairpin-deletion & hairpin & YHL5150 & pHL1150-1 & G7C & PBS \\
\hline YHL5037 & pHL1119-2 & purines to pyrimidines & hairpin & YHL5160 & pHL1 155-1 & G7U & PBS \\
\hline YHL5040 & pHL 1121-2 & pyrimidines to purines & hairpin & YHL5110 & pHL1 130-1 & U8G & PBS \\
\hline YHL4273 & pHL901-4 & G12A & 1 st ORF & YHL5116 & pHL1 133-1 & U8A & PBS \\
\hline YHL4285 & pHL902-4 & $\mathrm{G} 25 \mathrm{C}$ & 2nd ORF & YHL5130 & pHL1 140-1 & A10U & PBS \\
\hline YHL4297 & pHL903-4 & $\mathrm{G} 12 \mathrm{~A} / \mathrm{G} 25 \mathrm{C}$ & both ORFs & YHL5104 & pHL1127-1 & A10G & PBS \\
\hline YHL2706 ${ }^{\mathrm{b}}$ & pHL789-1 & $\mathrm{C} 176 \mathrm{G}$ & PBS & YHL5136 & pHL1 143-1 & $\mathrm{U} 11 \Delta$ & PBS \\
\hline YHL2722 ${ }^{\mathrm{b}}$ & pHL791-1 & G178C & PBS & YHL5019 & pHL1099-2 & U11A & PBS \\
\hline YHL2738 & pHL793-1 & C5G & PBS & YHL5154 & pHL1 152-1 & $\mathrm{A} 13 \mathrm{G}$ & $5-\mathrm{bp}$ \\
\hline YHL2754 ${ }^{\mathrm{b}}$ & pHL 795-1 & $\mathrm{G} 7 \mathrm{C}$ & PBS & YHL5020 & pHL1101-2 & G14C & 5-bp \\
\hline YHL $2770^{\mathrm{b}}$ & pHL789-1 & $\mathrm{G} 7 \mathrm{C} / \mathrm{C} 176 \mathrm{G}$ & PBS & YHL5144 & pHL1 147-1 & $\mathrm{C} 15 \mathrm{~A}$ & 5-bp \\
\hline YHL2786 & pHL799-1 & C5G/G178C & PBS & YHL5112 & pHL1131-1 & $\mathrm{C} 15 \mathrm{U}$ & $5-b p$ \\
\hline YHL5029 & pHL1 106-1 & Al12C & 11-bp & YHL5124 & pHL1137-1 & U16C & $5-b p$ \\
\hline YHL5025 & pHLl 104-1 & G109C & $11-b p$ & YHL5680 & pHL1245-1 & $\mathrm{C} 170 \mathrm{G}$ & bulge \\
\hline YHL5021 & pHL1102-1 & G105C & $11-b p$ & YHL5676 & pHL1244-1 & $\mathrm{A} 171 \mathrm{C}$ & bulge \\
\hline YHL5031 & pHL1 107-4 & $\mathrm{A} 112 \mathrm{C} / \mathrm{U} 184 \mathrm{G}$ & $11-b p$ & YHL5224 & pHL1195-1 & $\mathrm{U} 177 \mathrm{~A}$ & PBS \\
\hline YHL5027 & pHL1105-1 & G109C/U187G & $11-b p$ & YHL5156 & pHL1153-1 & U177G & PBS \\
\hline YHL5023 & pHL1103-2 & G105C/C191G & $11-b p$ & YHL5164 & pHL1157-1 & $\mathrm{U} 177 \mathrm{C}$ & PBS \\
\hline YHL5672 & pHL1243-1 & G165U & $5-b p$ & YHL5146 & pHL1148-1 & G178A & PBS \\
\hline YHL5684 & pHL1246-1 & $\mathrm{C} 15 \mathrm{~A} / \mathrm{G} 165 \mathrm{U}$ & $5-b p$ & YHL5132 & pHL1141-1 & G178U & PBS \\
\hline YHL5041 & pHL1122-1 & A216U & hairpin & YHL5126 & pHL1138-1 & $\mathrm{C} 181 \mathrm{U}$ & PBS \\
\hline YHL5328 & pHL1209-1 & $\mathrm{D} 564 \mathrm{~N}$ & $\mathrm{RT}$ & YHL5102 & pHL1126-1 & U184A & 11-bp \\
\hline YHL5337 & pHL1213-2 & U8G-D564N & PBS & YHL5114 & pHL1132-1 & U184G & $11-b p$ \\
\hline YHL5333 & pHL1211-2 & A10G-D564N & PBS & YHL5162 & pHL1156-1 & U185A & $11-b p$ \\
\hline YHL5252 & pHL1200-2 & U11A-D564N & PBS & YHL5152 & pHL1151-1 & U185G & $11-b p$ \\
\hline YHL5344 & pHL1217-1 & $\mathrm{U} 11-\Delta 564 \mathrm{~N}$ & PBS & YHL5106 & pHL1128-1 & U185C & $11-b p$ \\
\hline YHL5347 & pHL1218-2 & A13G-D564N & 5-bp & YHL5142 & pHL1146-1 & G186C & $11-b p$ \\
\hline YHL5231 & pHL1198-1 & G14C-D564N & $5-b p$ & YHL5228 & pHL1197-1 & G186U & 11-bp \\
\hline YHL5253 & pHL1201-1 & C15A-D564N & $5-b p$ & YHL5108 & pHL1129-1 & $\mathrm{Ul} 87 \mathrm{G}$ & $11-b p$ \\
\hline YHL5339 & pHL1214-2 & C15U-D564N & $5-b p$ & YHL5122 & pHL1136-1 & G188U & $11-b p$ \\
\hline YHL5341 & pHL1215-2 & U16C-D564N & 5-bp & YHL5148 & pHL1149-1 & A189C & $11-b p$ \\
\hline YHL5233 & pHL1199-1 & U177G-D564N & PBS & YHL5118 & pHLI134-1 & U190G & $11-b p$ \\
\hline YHL5331 & pHL1210-2 & U184A-D564N & 11-bp & YHL5140 & pHL1145-1 & U190A & $11-b p$ \\
\hline YHL5334 & pHL1212-1 & U187G-D564N & $11-b p$ & YHL5166 & pHL1158-1 & C191G & $11-b p$ \\
\hline YHL5255 & pHL1202-1 & C191G-D564N & $11-b p$ & YHL5120 & pHL1135-1 & C191A & $11-b p$ \\
\hline
\end{tabular}

${ }^{a}$ All plasmids were transformed into YHL912 (h-, ura4-294, leu1-32).

${ }^{\text {b }}$ Strains constructed by Levin (1996).

plasmid used in the experiments described in this report and plasmid pHL900-1 was used in each experiment as a negative control for transposition and reverse transcription. Plasmids pHL891-19 and pHL900-1 were constructed by the insertion of a SpeI-containing PCR-generated XhoI-AvrII fragment of $1.0 \mathrm{~kb}$ into pHL888-3 and pHL889-1, respectively. Plasmids pHL888-3 and pHL889-1 were constructed by inserting a PCR amplified neo fragment, which contained flanking $\mathrm{BamHI}$ sites and a silent mutation that destroyed a XhoI site, into the BglII site of pHL411-62 and pHL412-63 (Levin et al. 1993), respectively. The neo fragment lacking the $X$ hoI site was derived from pHL 765-3. Plasmid pHL411-62 contained the nmt1 promoter fused to Tf 1 at the start of the transposon transcript. The construct pHL41263 also was identical to pHL411-62 except for a frameshift mutation inserted at the Sacl site found in the beginning of PR. Plasmid pHL414-2 (Levin et al. 1993) resulted from the insertion of a neo gene into the BgIII site of pHL411-62. Plasmid pHL431-
25 was identical to pHL414-2 except that a reading frameshift in the beginning of IN was created. pH431-25 was constructed by ligating the $5.2-\mathrm{kb} X$ hol fragment, which contained Tf1, of pHL338-9 (Levin 1995) into the $8.5 \mathrm{~kb}$ Xhol fragment, which constituted the vector, of pHL414-2.

The construction and amplification of a plasmid library that contained mutations in $T f 1$

The plasmid library containing mutations in the $5^{\prime}$ UTR of Tf 1 was constructed by cloning a PCR-amplified fragment of $230 \mathrm{bp}$ into the unique XhoI and SpeI sites of pHL891-19. Two oligonucleotides, HL100 and HL101, were made degenerate by the addition of all 4 nucleotides during the oligonucleotide synthesis at a concentration 250 -fold less than the normal level for the wild-type nucleotide. Using these two oligonucleotides, the 230-bp XhoI-SpeI fragment was PCR amplified and ligated into 
$\frac{9}{\frac{9}{m}} \frac{\stackrel{5}{\circ}}{\circ}$

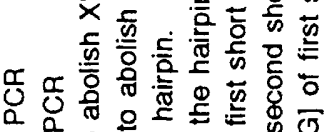

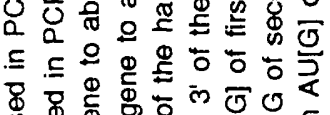

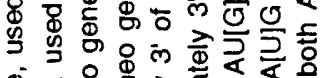

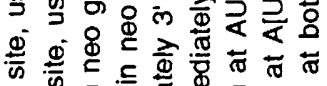

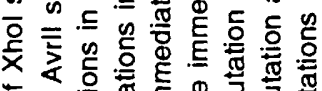

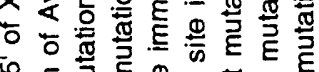

\& $\overline{0}$ ह

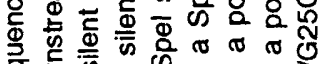

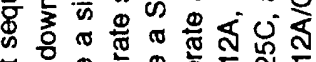

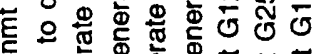

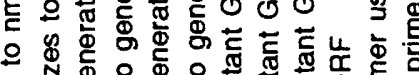

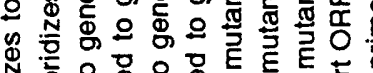

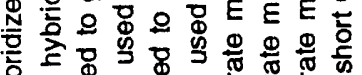

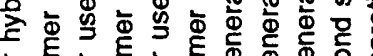

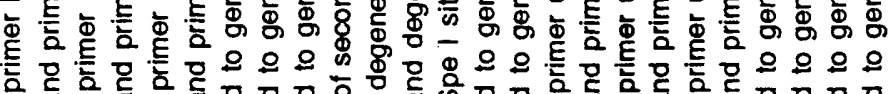

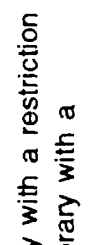

突毫

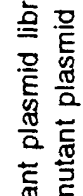

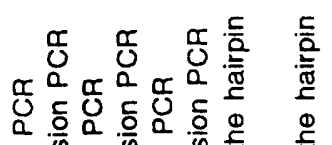

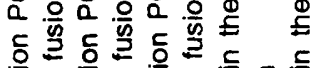

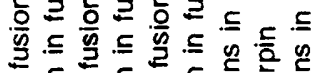

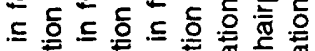

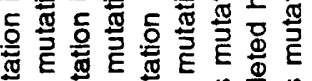

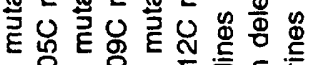

สั

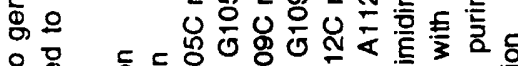

요용

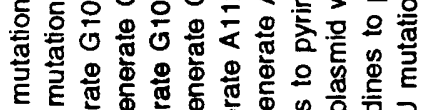

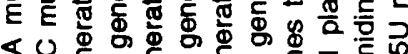

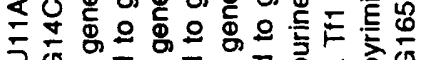

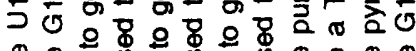

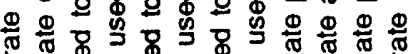

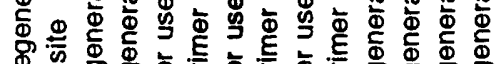

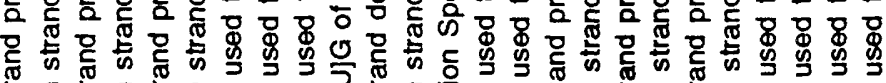

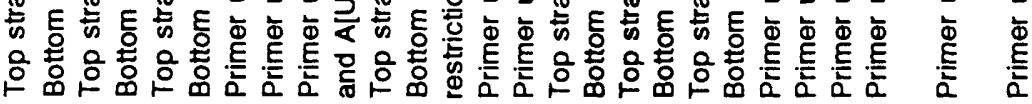
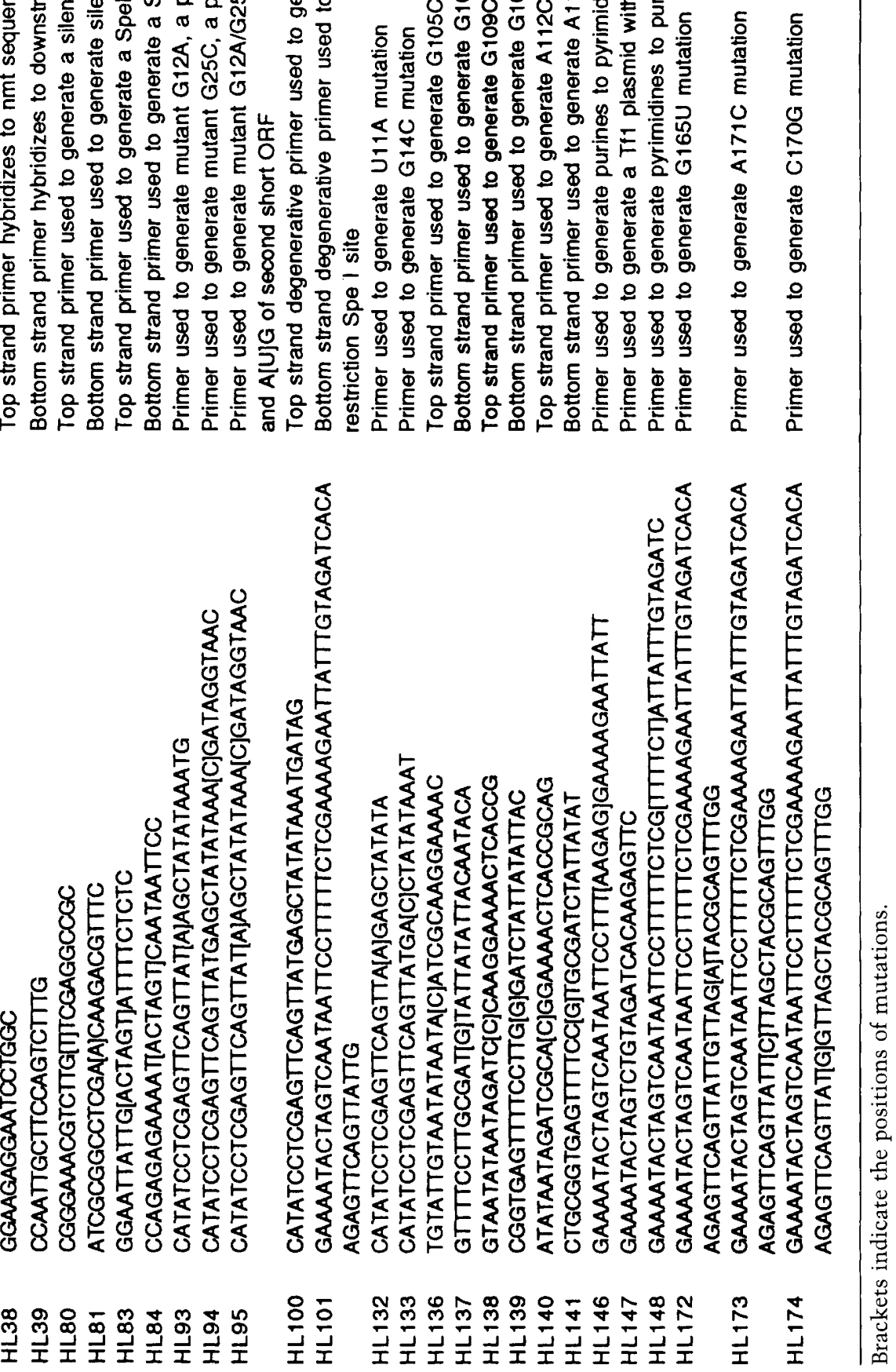
pHL891-19. The ligated products were electroporated into ElectroMax DH1OB cells (Life Tech. Inc.).

Recovery of plasmids from S. pombe strains with defective Tf1 transposition

The mutant plasmids were transformed into YHL912. Five thousand transformed colonies were subjected to transposition patch assays. The cells identified as defective for transposition were harvested from $10-\mathrm{ml}$ cultures grown to an $\mathrm{OD}_{600}$ of 2.0 in EMM minus uracil dropout media that contained $10 \mu \mathrm{M}$ thiamine. The cells were resuspended in $1.5 \mathrm{ml}$ of $2 \mathrm{mg} / \mathrm{ml} \mathrm{Zy-}$ molyase $20 \mathrm{~T}$ (ICN) in a buffer consisting of $50 \mathrm{mM} \mathrm{Na}_{2} \mathrm{HPO}_{4}, 50$ $\mathrm{mm}$ citric acid, and $1.2 \mathrm{M}$ sorbitol at $\mathrm{pH} 5.6$ and incubated at $37^{\circ} \mathrm{C}$ for $1 \mathrm{hr}$. The enzyme-treated cells were pelleted and resuspended in $300 \mu \mathrm{l}$ of TE. Thirty-five microliters of $10 \%$ SDS was added and the mixture was incubated at $65^{\circ} \mathrm{C}$ for $5 \mathrm{~min}$. After addition of $100 \mu \mathrm{l}$ of $5 \mathrm{M} \mathrm{KOAc}$ and incubation on ice for $30 \mathrm{~min}, 400 \mu \mathrm{l}$ of supernatant was collected from a 10-min microcentrifuge spin at full speed. The nucleic acids in the supernatant were bound to $30 \mu \mathrm{l}$ of Prep-a-gene matrix (Bio-Rad) in $1.2 \mathrm{ml}$ of binding buffer and eluted with $50 \mathrm{ml}$ of TE after washing with $2 \mathrm{ml}$ of washing buffer. The XhoI-SpeI fragment from each mutant strain identified was sequenced to identify base changes. The 5' UTR segment of Tf 1 from plasmids isolated by the mutant screen was subcloned into pHL891-19 as a 3794-bp MluI-SpeI fragment to ensure that no mutations created during the initial yeast transformation existed in Tfl outside of the region between $\mathrm{XhoI}$ and SpeI.

\section{Construction of plasmids with site-directed mutations}

The plasmids containing site-directed mutations, including the single- and multiple-point mutations and deletions, were constructed by cloning into pHL891-19 the XhoI-SpeI fragment amplified with oligonucleotides that included the specific mutations. The plasmids containing compensating double-point mutations in the 5' UTR of Tf1, including plasmids pHL1103-1, $1105-1,1107-1$, and 1246-1, were created by using DNA from the plasmids with single-point mutation as template in either regular or fusion PCR reactions that used the corresponding oligonucleotides to generate the additional mutation. The plasmids with double mutations that contained D564N in RT were constructed by subcloning the $8.25-\mathrm{kb} A v r I I-B a m H I$ fragment from the mutant plasmids into the 5.45-kb AvrII-BamHI fragment of pHL944-1 (Levin 1996).

\section{Particle preparation and extraction of nucleic acid for -ssDNA detection}

The preparations of large-scale yeast extracts and the subsequent analysis on sucrose gradients was based on previously published protocols (Garfinkel et al. 1985; Eichinger and Boeke 1990). Cells were inoculated at an $\mathrm{OD}_{600}$ of $0.05 \mathrm{in} 500 \mathrm{ml}$ of EMM minus uracil drop out media and harvested at an $\mathrm{OD}_{600}$ of 1.0. (Levin et al. 1993). Nucleic acid was extracted from sucrose fractions containing virus-like particles. -ssDNA was detected according to a previously published protocol (Levin 1995).

\section{Isolation of RNA and DNA from whole-cell extracts}

Ten milliliters of stationary-phase cells at $\mathrm{OD}_{600}$ and 10 or 50 $\mathrm{ml}$ of log-phase cells at $\mathrm{OD}_{600}$ of 2.0 were harvested for nucleic acids isolation. These preparations, as described previously (Levin 1996), were used to generate materials for either DNA blot analysis (Atwood et al. 1996) or RNA-templated primer extension.

\section{Primer extension}

One-tenth of the isolated RNA $(\sim 10 \mu \mathrm{g})$ was resuspended in 15 $\mu \mathrm{l}$ of $1 \times$ AMV reverse transcription buffer (Boehringer Mannheim) that contained 1 pmole of $5^{\prime}$ end- ${ }^{32} \mathrm{P}$-labeled oligonucleotide JB890. Extension reactions with AMV RT (Boehringer Mannheim) were as described previously (Levin 1996).

\section{Acknowledgments}

We acknowledge the support of the National Institutes of Health Intramural AIDS Targeted Antiviral Program.

The publication costs of this article were defrayed in part by payment of page charges. This article must therefore be hereby marked "advertisement" in accordance with 18 USC section 1734 solely to indicate this fact.

\section{References}

Aiyar, A., D. Cobrinik, Z. Ge, H.J. Kung, and J. Leis. 1992. Interaction between retroviral U5 RNA and the T psi C loop of the tRNA(Trp) primer is required for efficient initiation of reverse transcription. I. Virol. 66: 2464-2472.

Aiyar, A., Z. Ge, and J. Leis. 1994. A specific orientation of RNA secondary structures is required for initiation of reverse transcription. J. Virol. 68: 611-618.

Atwood, A., J. Lin, and H. Levin. 1996. The retrotransposon Tf1 assembles virus-like particles with excess Gag relative to integrase because of a regulated degradation process. Mol. Cell. Biol. 16: 338-346.

Boeke, J.D., J. Trueheart, G. Natsoulis, and G.R. Fink. 1987. 5-Fluoro-orotic acid as a selective agent in yeast molecular genetics. Methods Enzymol. 154: 164-175.

Chapman, K.B., A.S. Byström, and J.D. Boeke. 1992. Initiator methionine tRNA is essential for Tyl transposition in yeast. Proc. Natl. Acad. Sci. 89: 3236-3240.

Donze, O. and P.F. Spahr. 1992. Role of the open reading frames of Rous sarcoma virus leader RNA in translation and genome packaging. EMBO J. 11: 3747-3757.

Eichinger, D.J. and J.D. Boeke. 1990. A specific terminal structure is required for Tyl transposition. Genes \& Dev. 4: 324 330.

Friant, S., T. Heyman, M.L. Wilhelm, and F.X. Wilhelm. 1996. Extended interactions between the primer tRNAi(Met) and genomic RNA of the yeast Tyl retrotransposon. Nucleic Acids Res. 24: 441-449.

Gabriel, A. and J.D. Boeke. 1993. Retrotransposon reverse transcription. In Reverse transcriptase (ed. S. Goff and A. Skalka), pp. 275-328. Cold Spring Harbor Laboratory Press, Cold Spring Harbor, NY.

Garfinkel, D.J., J.D. Boeke, and G.R. Fink. 1985. Ty element transposition: Reverse transcriptase and virus-like particles. Cell 42: 507-517.

Keeney, J.B., K.B. Chapman, V. Lauermann, D.F. Voytas, S.U. Astrom, U. von Pawel-Rammingen, A. Byström, and J.D. Boeke. 1995. Multiple molecular determinants for retrotransposition in a primer tRNA. Mol. Cell. Biol. 15: $217-$ 226.

Kikuchi, Y., Y. Ando, and T. Shiba. 1986. Unusual priming mechanism of RNA-directed DNA synthesis in copia retrovirus-like particles of Drosophila. Nature 323: 824-826.

Kohlstaedt, L., J. Wang, J. Friedman, P. Rice, and T. Steitz. 1992. 
Crystal structure at $3.5 \AA$ resolution of HIV-1 reverse transcriptase complexed with an inhibitor. Science 256: 17831790.

Leis, J., A. Aiyar, and D. Cobrinik. 1993. Regulation of initiation of reverse transcription of retroviruses. In Reverse transcriptase (ed. A. Skalka and S. Goff), pp. 33-47. Cold Spring Harbor Laboratory Press, Cold Spring Harbor, NY.

Levin, H.L. 1995. A Novel mechanism of self-primed reverse transcription defines a new family of retroelements. Mol. Cell. Biol. 15: 3310-3317.

- 1996. An unusual mechanism of self-primed reverse transcription requires the RNase $\mathrm{H}$ domain of reverse transcriptase to cleave an RNA duplex. Mol. Cell. Biol. 16: 56455654.

Levin, H.L. and J.D. Boeke. 1992. Demonstration of retrotransposition of the $\mathrm{Tf} 1$ element in fission yeast. EMBO $J$. 11: $1145-1153$.

Levin, H.L., D.C. Weaver, and J.D. Boeke. 1990. Two related families of retrotransposons from Schizosaccharomyces pombe. Mol. Cell. Biol. 10: 6791-6798.

Levin, H.L., D.C. Weaver, and J.D. Boeke. 1993. Novel gene expression mechanism in a fission yeast retroelement: Tf1 proteins are derived from a single primary translation product [published erratum appears in EMBO J. 13: 1494 (1994)]. EMBO J. 12: 4885-4895.

Moreno, S., A. Klar, and P. Nurse. 1991. Molecular genetic analysis of fission yeast Schizosaccharomyces pombe. Methods Enzymol. 194: 795-823.

Moustakas, A., T.S. Sonstegard, and P.B. Hackett. 1993a. Alterations of the three short open reading frames in the Rous sarcoma virus leader RNA modulate viral replication and gene expression. J. Virol. 67: 4337-4349.

- 1993b. Effects of the open reading frames in the Rous sarcoma virus leader RNA on translation. J. Virol. 67: 43504357.

Varmus, H.E. and R. Swanstrom. 1984. Replication of retroviruses. In RNA tumor viruses (ed. R. Weiss, N. Teich, $\mathrm{H}$. Varmus, and J. Coffin), pp. 369-512. Cold Spring Harbor Laboratory, Cold Spring Harbor, NY.

Wilhelm, M., F.X. Wilhelm, G. Keith, B. Agoutin, and T. Heyman. 1994. Yeast Ty1 retrotransposon: The minus-strand primer binding site and a cis-acting domain of the Tyl RNA are both important for packaging of primer tRNA inside virus-like particles. Nucleic Acids Res. 22: 4560-4565. 


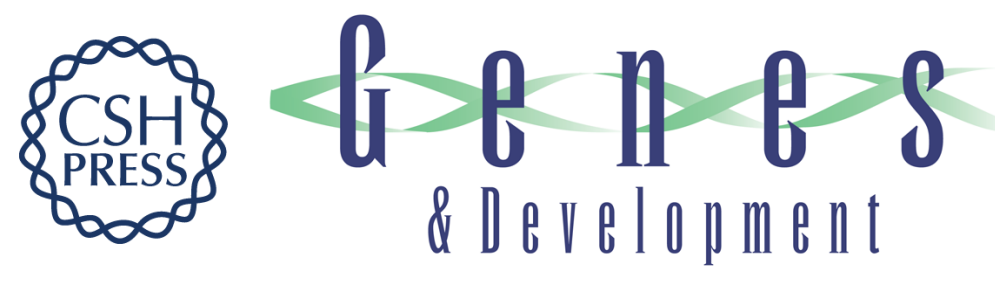

\section{A complex structure in the mRNA of Tf1 is recognized and cleaved to generate the primer of reverse transcription.}

$\mathrm{J} \mathrm{H}$ Lin and $\mathrm{H} \mathrm{L}$ Levin

Genes Dev. 1997, 11:

Access the most recent version at doi:10.1101/gad.11.2.270

References This article cites 21 articles, 12 of which can be accessed free at: http://genesdev.cshlp.org/content/11/2/270.full.html\#ref-list-1

License

Email Alerting Service right corner of the article or click here.

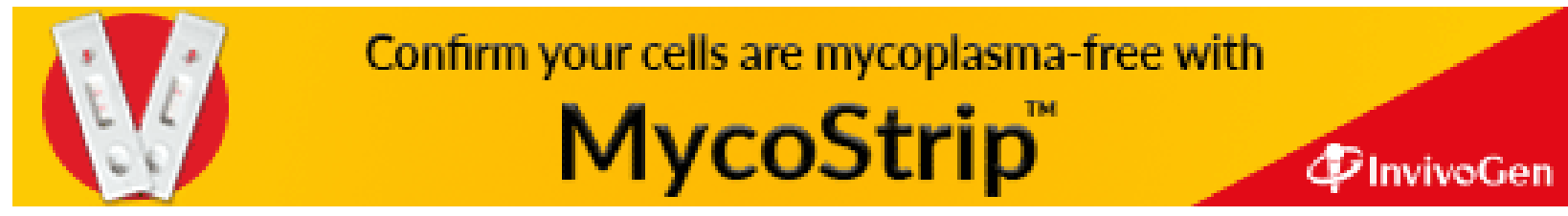

